\title{
Ontologization of tools for modeling and synthesis technological process trajectories
}

\author{
Vasily Mokhov ${ }^{1}$, Danil Shaykhutdinov ${ }^{1, *}$, Evgeniy Kirievskiy ${ }^{1}$, Alexander Vlasov $^{1}$, and \\ Nikolay Gorbatenko ${ }^{1}$ \\ ${ }^{1}$ Platov South-Russian State Polytechnic University (NPI), Prosveshcheniya Street, 132, \\ Novocherkassk, 346428, Russia
}

\begin{abstract}
The publication is devoted to the development of an ontological model of tools for intelligent modeling and synthesis of the trajectories of technological processes in electric power networks. Development includes two levels of modeling: the level of metaontology and the level of applied ontologies. At the upper level, the structure of the upper level is proposed and described. At the level of applied ontologies, the ontology of the "digital substation - distribution network" system and the structure for the ontology of tasks are detailed. The first is implemented and presented in the Protégé framework environment. The second is presented descriptively by the example of the task of minimizing energy losses at the level of a transformer substation with an illustration of a functional diagram of the technological chain of its solution. The findings of the work reflect the potential of using the proposed solution to prepare an integrated knowledge management system in the subject area.
\end{abstract}

\section{Introduction}

The current moment of the historical development of the productive forces of mankind is the fourth industrial revolution [1]. In a broad sense, Industry 4.0 characterizes the current trend in the development of automation and data exchange, which includes cyber-physical systems, the Internet of Things and cloud computing.

The essence of the next upcoming revolution is that the material world must connect with the virtual one, as a result of which new cyberphysical complexes will appear, combined into a single digital system. Industry 5.0, based on the integration of the Internet of Knowledge and the Internet of Things, should enable a non-programming knowledge carrier to enter them into a computer without intermediaries. It became possible with the advent of expert programming methodology, in which knowledge is described in the language of business prose, as close as possible to the literary language, but formalized so that it is possible to automatically generate software tools that correspond to the source texts. The basis of this technology is the Internet of Knowledge, which is built on the basis of ontologies and metaontologies [2, 3].

\footnotetext{
*Corresponding author: d.shaykhutdinov@npi-tu.ru
} 
As part of the implementation of the departmental project of the Ministry of Energy of Russia "Digital Energy" (https://minenergo.gov.ru/node/14559) in terms of reducing technological disruptions and accidents at electric power facilities, formalization and accumulation of knowledge obtained during the development and study of tools for intelligent modeling and synthesis of the trajectories of technological processes of electric power grids are actively gaining importance [4-6] at the level of ontological models.

The practical representation of knowledge models at the ontological level is widely used. Thus, the IEEE Power Engineering Society (IEEE) Subcommittee on Intelligent System Applications ISA (Intelligent System Applications) is studying the development and application of methodologies and tools for intelligent systems to solve problems in the energy field. This subcommittee has been publishing and promoting the use of open data sets for energy consumption, electric vehicles, electric power quality, general energy data, etc., for scientific purposes for about ten years (https://site.ieee.org/pes-iss/data-sets/\#reli). The latter is provided to users in RDF formats based on the ontology import of the ITEA2 12004 SEAS project (Smart Energy Aware Systems) [7], which once again confirms the relevance of formalizing and presenting knowledge based on the ontological approach.

\section{Problem formulation}

The task is to develop an ontological model (formalized electronic model of knowledge) of means of intellectual modeling and synthesis of the trajectories of technological processes in electric power networks. In accordance with the main ideas of ontological modeling [8, 9], the goals of the subsequent application of the developed model include:

1) ensuring accessibility for perception and exchange of structured information about the subject area between specialists;

2) performing simulation of these processes in order to optimize them;

3) prompt receipt of conclusions based on a large amount of information, in order to support decision-making;

4) solving technical problems in the field of integration of information systems.

The key object for formalizing the description is the electric energy distribution system, which is a combination of "digital substation - distribution network" (DS-DN). The distribution network includes distribution transformer substations (TS), power lines connecting them to a digital substation (DS), as well as many single-phase consumers of electricity (SPC) receiving electricity from the TS.

Under the technological process we consider the process of transmission and distribution of electric power to SPC and the associated tasks of increasing its efficiency by reducing the loss of electricity caused by harmonic components of the current.

\section{Materials and methods}

As it is known, the formed ontologies make it possible to efficiently analyze conceptual models at the level of designs, since they are openly expressed detailed classifications of sets of essential features of simulated systems [10]. Moreover, ontological modeling is based on three stages [11]:

1) compilation of a dictionary of concepts;

2) definition of the relationship between concepts;

3) preparation of a set of options for interpreting the results of paragraph 1 and paragraph 2 .

Ontological modeling begins with an ontological analysis of the subject area, specifically with the compilation of a glossary of terms, which is used to study the 
characteristics of the system and processes that make up the subject area, as well as creating a database of definitions of these terms.

Any system is characterized by at least two categories of objects of perception: directly the constituent objects and the relationships between them (characterizing the state of the system). Therefore, at the second stage the basic logical relationships between the relevant terms and concepts are documented. The result of this analysis is the ontology of the system or a glossary, providing exact definitions and the relationships between the terms.

The presentation of the ontology only at the level of paragraphs 1 and 2 is often insufficient to understand without appropriate examples of use. Therefore, in practice, to represent the ontology, many concepts and relations between them, as a rule, are supplemented by a set of options for interpreting the specified set using specific examples.

Formally, an ontology is defined as an ordered triple of the form [12]:

$$
\mathbf{O}=<\mathbf{X}, \mathbf{R}, \mathbf{F}>\text {, }
$$

where $\mathbf{X}$ is a finite set of concepts (terms) of the domain that $\mathbf{O}$ ontology represents; $\mathbf{R}$ is a finite set of relations between concepts of a given subject area; $\mathbf{F}$ - a finite set of interpretation functions defined in the concepts and / or relations of $\mathbf{O}$ ontology.

In Russia, a significant contribution to the development of the ideas of ontological modeling was made by T.A. Gavrilova. In the works of this author, it is also noted that ontological engineering is a powerful tool for structuring knowledge and includes the following steps [13]:

- allocation of basic concepts of the subject area;

- definition of "height of the ontology tree" the number of levels of abstraction;

- distribution of concepts by levels;

- determining the relationships and interactions of basic concepts;

- consultations with various specialists to eliminate contradictions and inaccuracies.

Regarding tools for working with ontologies, the leading position is held by the Stanford development Protégé, a freeware editor of open source ontologies, which at the same time serves as a structure for creating intelligent systems [14, 15]. Moreover, as noted on the official website of Protégé, "it is supported by a strong community of academic, government and corporate users who use it to create knowledge-based solutions" (https://protege.stanford.edu) in various subject areas.

\section{Proposed decision}

\subsection{Metaontology level}

At the metaontology level, it is customary to operate within general concepts and relationships that are independent of a specific subject area. In accordance with this, at the highest level of abstraction, we will use a structure that includes two main components: objective ontology and ontology of tasks (Fig. 1). Moreover, the latter will be represented by task ontologies of analysis, synthesis, optimization, and equipment (technological and technical). 


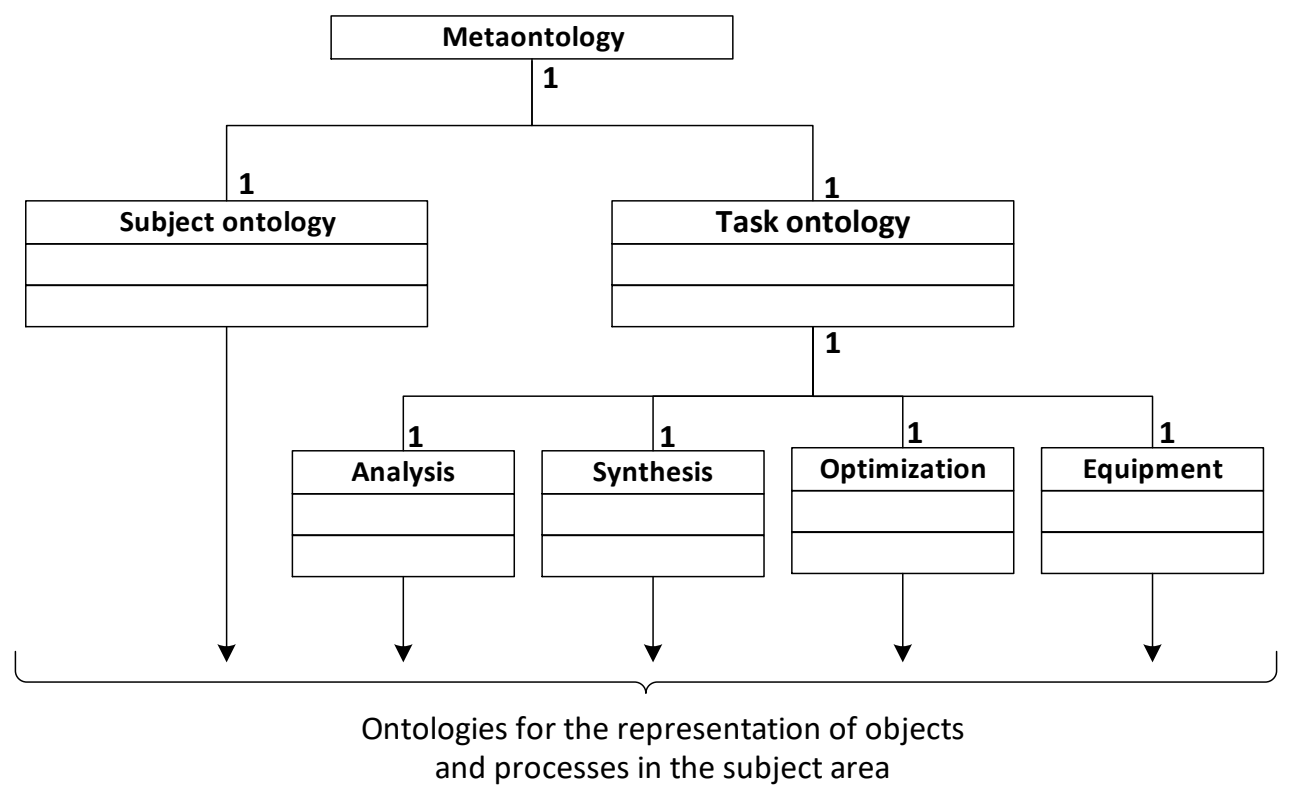

Fig. 1. The structure of formalization of knowledge at the level of metaontology.

Each of the listed components should be formalized at the level of the corresponding ontology of the applied level for representing objects and processes of the subject area.

\subsection{Subject ontology}

As an ontology of the application level, we illustrate the ontology of the system "digital substation - distribution network" in the form of a taxonomic hierarchy of classes corresponding to the above description of the subject area (Fig. 2).

The root object of this ontology is digital substation. This object has two sets of characteristics necessary for research and solving problems of reducing energy losses [4, 5]. The first of them is determined at the level of such passport characteristics as higher voltage, lower voltage, current on the higher voltage side, current on the lower voltage side. The second set of characteristics is determined by the dynamic parameters of the transformer in the following composition: rated higher voltage, open circuit current, short circuit voltage, open circuit loss and short circuit loss.

A digital substation manages a distribution network that has a specific structure (trunk, radial, ring or mixed) of consumer connections between TS (at least one) and power line characteristics.

In this case, TS, as well as a digital substation, is determined by the dynamic parameters of the transformer similar in composition. Passport characteristics in comparison with a digital substation are supplemented by detailed characteristics for the parameters of the equivalent circuit. In the case of the L-shaped equivalent circuit, the calculations use data on the characteristics of the resistances of the longitudinal and transverse branches, in the case of T-shaped circuits the data on the characteristics of the resistances of the windings of higher and lower voltage, as well as the resistance of the magnetic circuit, is used. 


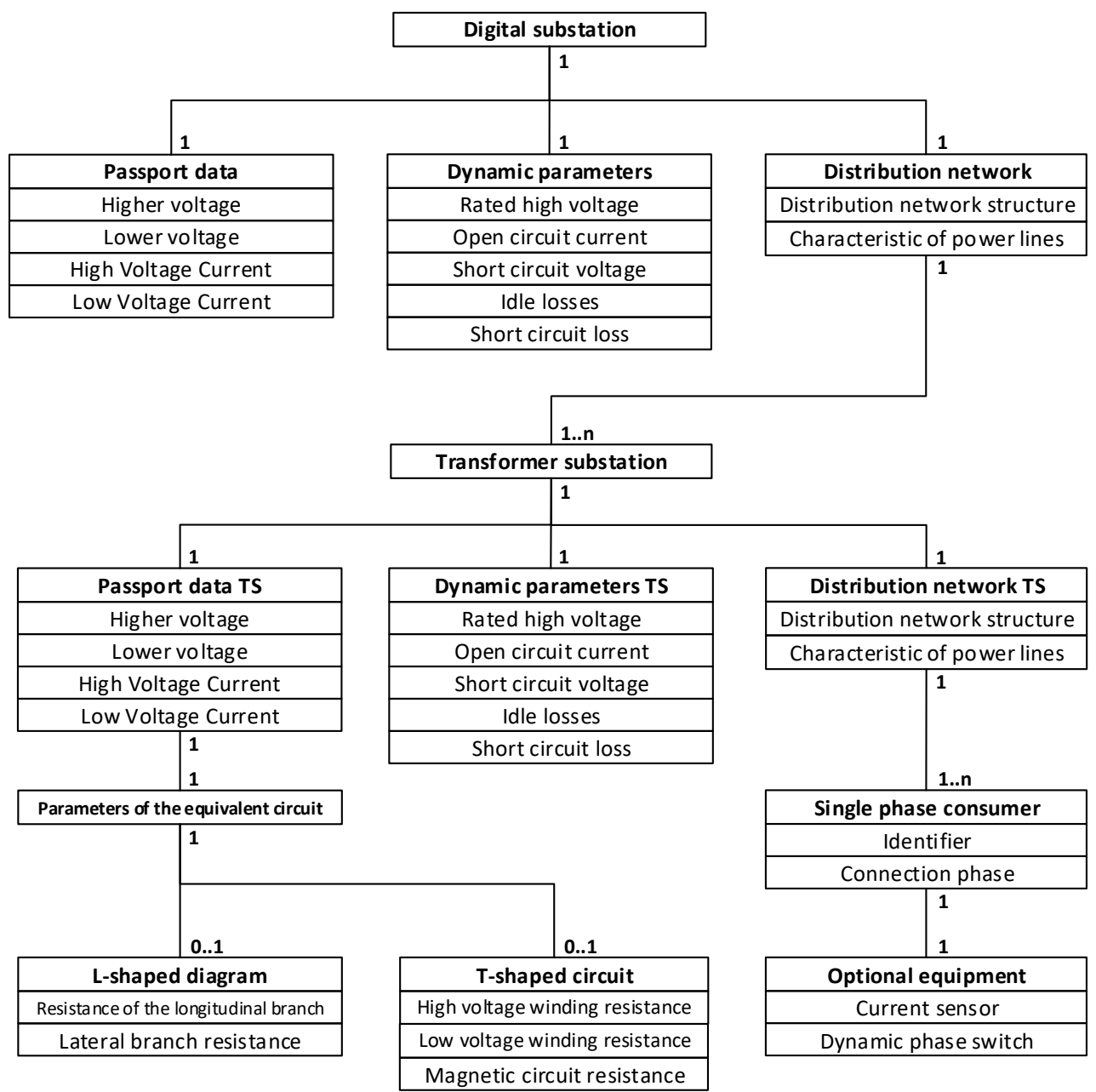

Fig. 2. The structure of formalization of knowledge at the level of subject ontology.

Distribution network of TS is characterized by the structure of consumer connections and the characteristic of power lines. Unlike the distribution network of a digital substation, here the consumers are SPC, each of which has a unique identifier (number) and the connection phase at the current time. Each SPC is provided with additional equipment, which should include a current sensor for generating digitized waveforms of the current consumption of the SPC and a means of dynamically switching the SPC in relation to the phases of the TS.

\subsection{Task ontology}

So, for example, for the task of minimizing energy losses at the TS level the functional diagram of the technological chain (model) of its practical solution is illustrated in Fig. 3. 




Fig. 3. Functional flowchart diagram for minimization of energy losses.

The initial data for this task is:

1)number of SPC connected to TS;

2)the initial combination of phase numbers for connecting the SPC to TS;

3)a set of digitized current waveforms for the SPC connected to TS.

Two methods of solution are required:

1)ensuring a symmetrical mode of operation of a three-phase electric power transmission system;

2)lowering the higher harmonic components in the spectral composition of the load current of the distribution TS due to their mutual suppression.

As a result of the decision, a new combination of the numbers of the SPC connections to the TS is formed. The remaining details of the solution to the problem are presented in [6].

\section{Discussion}

Specifically for the "digital substation - distribution network" system in accordance with the structure shown in Fig. 2, the authors developed an ontological model. The ontology was prepared using the Protégé editor version 5.5.0, created by the Stanford Center for Biomedical Computer Science Research. A fragment of the implementation of the proposed solution is illustrated in Fig. 4. 


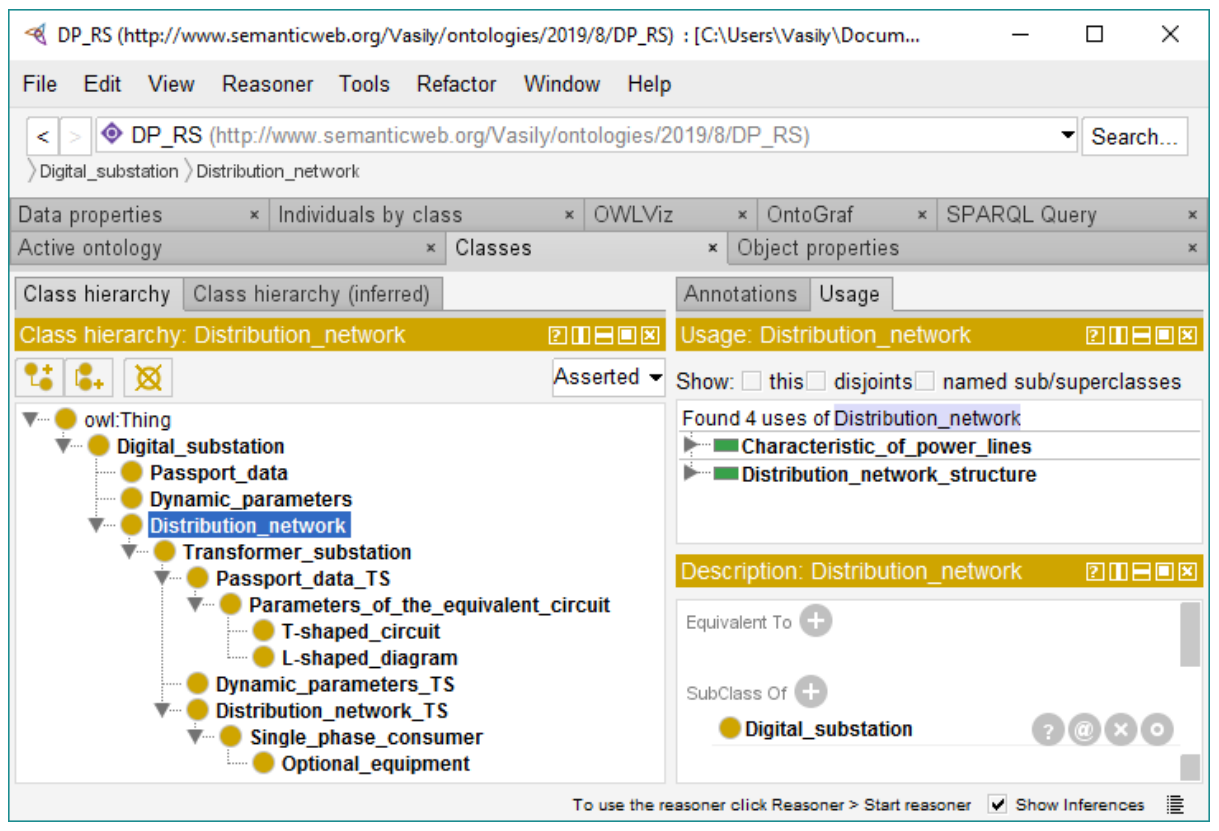

Fig. 4. A fragment of the ontological model "digital substation - distribution network" in Protégé.

In accordance with the objectives of the work and according to the proposed solutions, it is planned in the future to prepare an integrated knowledge management system for the means of intellectual modelling and synthesis of the trajectories of technological processes of electric power networks.

\section{Conclusion}

The approach described in the work significantly simplifies the accumulation and analysis of knowledge in the subject area under consideration and makes it possible to reuse it. As a result, competencies are formed from knowledge, which, in turn, serve as the basis for creating new and improving existing technical solutions. In addition, the development of an appropriate knowledge management system will provide control over the implementation of the processes of formation and exchange of new knowledge, its use in decision making, the application of knowledge in products and services, as well as providing access for specialists to knowledge of the subject area.

This research was funded by RFBR, project number 18-38-20188.

\section{References}

1. V. Tarasov, Strat. Dec. \& Risk Mang. 2(107), 62-69 (2018) DOI:10.17747/20788886-2018-2-62-69

2. G.B. Yevgenev, Ont. of Des. 9(31), 7-23 (2019) DOI: 10.18287/2223-9537-2019-9-17-23

3. L. Yang, K. Cormican, M. Yu, Comput. Ind. 111, 148-171 (2019)

4. S.S. Kostinskiy, V.A. Mokhov, T.N. Kruglova, D.V. Shaikhutdinov, A.S. Vlasov, ISPC Conf. Ser.: Mat. Sci. \& Eng. 483(1), 012080 (2019) 
5. V.A. Mokhov, Univ. News. North-Cauc. Reg. Tech. Sci. Ser., 1, 51-56 (2019) DOI: 10.17213/0321-2653-2019-1-51-56

6. V. Mokhov, S. Kostinskiy, et al., Adv. Int. Sys. \& Comp., 983, 611-618 (Springer, Cham, 2018)

7. M. Lefrançois, J. Kalaoja, T. Ghariani, A. Zimmermann, The SEAS Knowledge Model in ITEA2 12004 Smart Energy Aware Systems (2017)

a. $\quad$ Eibeck, M.Q. Lim, M. Kraft, Comput. Chem. Eng. 131, 106586 (2019)

8. V. Zaletelj, E. Hozdic, P. Butal, Adv. Eng. Inf. 38, 129-141 (2018)

9. Y. Wand, V.C. Storey, R. Weber, ACM Trans. on Datab. Sys. (TODS) 24(4) 494-528 (1999)

10. M.C. Suarez-Figueroa, A. Gomez-Perez, M. Fernández-López, Data Know. Eng. 102, 1-21 (2016)

11. T. Gavrilova, I. Leshcheva, E. Strakhovich, Know. Manage. Res. Pract. J. 13(4), 418428 (2015)

12. T. A. Gavrilova, J. Know. Manage. 16(4), 523-537 (2012)

13. M. Vigo, N. Matentzoglu, C. Jay, R. Stevens, J. Web Sem. 57, 100473 (2019)

14. P. Rodler, D. Jannach, K. Schekotihin, P. Fleiss, Know.-Based Syst. 179, 92-107 (2019) 